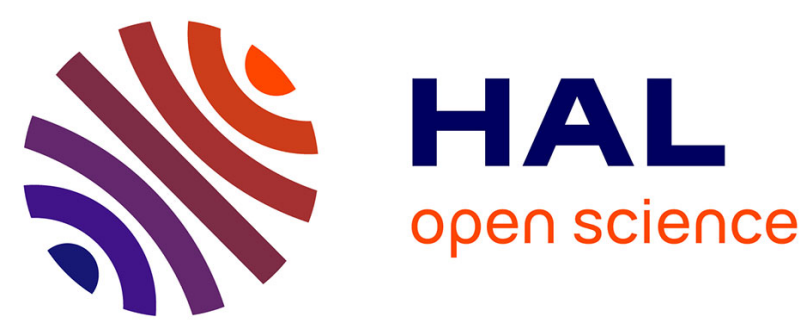

\title{
Efficacy of local attack strategies on the Beijing road complex weighted network
}

\author{
M. Bellingeri, Danièle Bevacqua, Francesco Scotognella, Zhe-Ming L., D. Cassi
}

\section{To cite this version:}

M. Bellingeri, Danièle Bevacqua, Francesco Scotognella, Zhe-Ming L., D. Cassi. Efficacy of local attack strategies on the Beijing road complex weighted network. Physica A: Statistical Mechanics and its Applications, 2018, 510, pp.316-328. 10.1016/j.physa.2018.06.127 . hal-02621283

\section{HAL Id: hal-02621283 \\ https://hal.inrae.fr/hal-02621283}

Submitted on 26 May 2020

HAL is a multi-disciplinary open access archive for the deposit and dissemination of scientific research documents, whether they are published or not. The documents may come from teaching and research institutions in France or abroad, or from public or private research centers.
L'archive ouverte pluridisciplinaire HAL, est destinée au dépôt et à la diffusion de documents scientifiques de niveau recherche, publiés ou non, émanant des établissements d'enseignement et de recherche français ou étrangers, des laboratoires publics ou privés.

\section{(ㅇ)(1) $\$$}

Distributed under a Creative Commons Attribution - NonCommercial - NoDerivatives| 4.0 


\section{Accepted Manuscript}

Efficacy of local attack strategies on the Beijing road complex weighted network

M. Bellingeri, D. Bevacqua, F. Scotognella, Zhe-Ming L., D. Cassi

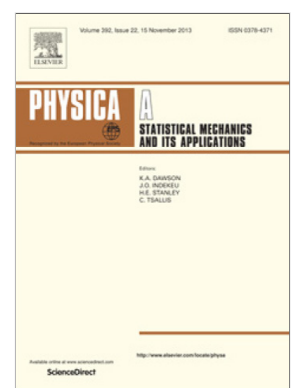

PII:

S0378-4371(18)30851-3

DOI: $\quad$ https://doi.org/10.1016/j.physa.2018.06.127

Reference: $\quad$ PHYSA 19821

To appear in: $\quad$ Physica A

Received date: 9 January 2018

Revised date: 8 June 2018

Please cite this article as: M. Bellingeri, D. Bevacqua, F. Scotognella, Z.-M.L.. Z.-M.L., D. Cassi, Efficacy of local attack strategies on the Beijing road complex weighted network, Physica A

(2018), https://doi.org/10.1016/j.physa.2018.06.127

This is a PDF file of an unedited manuscript that has been accepted for publication. As a service to our customers we are providing this early version of the manuscript. The manuscript will undergo copyediting, typesetting, and review of the resulting proof before it is published in its final form. Please note that during the production process errors may be discovered which could affect the content, and all legal disclaimers that apply to the journal pertain. 


\section{Highlights}

- we produce local attacks on the Bejing road complex weighted network,

- the efficacy of the attack strategies changed using binary or weighted measures.

- removing nodes according to weighted rank produced the higher damage;

- to find important nodes it is necessary to account the weight of the links.

- a non-negligible fraction of random removals is more efficient than the best attack strategies;

- to find the most important nodes may be more efficient to simulate an higher number of random node removals. 


\title{
Efficacy of local attack strategies on the Beijing road complex
}

\section{weighted network}

Bellingeri M. ${ }^{1 *}$, Bevacqua D. ${ }^{2}$, Scotognella F. ${ }^{3,4}$, Zhe-Ming L. ${ }^{5}$, Cassi D. ${ }^{1}$

${ }^{1}$ Dipartimento di Fisica, Università di Parma, via G.P. Usberti, 7/a, 43124 Parma, Italy

* Corresponding author: michele.bellingeri@nemo.unipr.it

${ }^{2}$ PSH UR 1115, INRA, 84000, Avignon, France

${ }^{3}$ Dipartimento di Fisica, Politecnico di Milano, Piazza Leonardo da Vinci 32, 20133 Milano, Italy

${ }^{4}$ Center for Nano Science and Technology@PoliMi, Istituto Italiano di Tecnologia, Via Giovanni Pascoli, 70/3, 20133, Milan, Italy

${ }^{5}$ School of Aeronautics and Astronautics, Zhejiang University, Hangzhou, 310027, P.R.China

\begin{abstract}
Understanding the complex network response to nodes failure is important to protect them and guarantee their functioning. Most existing studies considered binary networks where the intensity (weight) of the links is not accounted for. However, in real world networks the intensity of the links widely differ so that a realistic description of complex networks is only possible if the heterogeneity present in edge weights is taken into account.
\end{abstract}

Further, the vast majority of the existing studies investigates the efficacy of the attack strategies when all the nodes are removed. Despite it is important to study the network behavior in such a circumstance, real-world networks are more likely to experience local failures involving a small amount of nodes with a decrease in the system functioning not causing an overall collapse. This is 
the case of crossroad closing in complex road networks. For this reason it is interesting to investigate the networks response focusing to small node removals.

In this paper we analyze the response of the real-world road networks of Beijing, the capital of China, to the removal of 1 and 2 nodes and accounting the weighted structure of the network. We use both classic binary node properties and network functioning measurement, introduced a weighted rank for node importance (node strength or page rank), and used a measurement for network functioning that accounts for the weight of the links (weighted efficiency).

We find that the weighted node attack strategy Str (i.e. strength attack), which removes nodes according to their strength, is the most efficient to blemish the system when evaluating the damage with a weighted efficiency. Differently, betweenness attack strategy Bet, which removes nodes according to their centrality is the most efficient when using the binary efficiency to compute the network damage. These results indicate that adopting weighted or binary efficiency to measure networks functioning changed the efficacy of the attack strategies to damage the system. Last, we found a non-negligible fraction of random removals to be more efficient than the attack strategies; given that the main goal of the attack strategies is to individuate important nodes in the system, i.e. nodes which removal triggers highest damage, our results would indicate how in order to find the most important nodes may be more efficient to simulate an higher number of random nodes removal.

\section{Introduction}

The response of complex networks to nodes failure (i.e. the removal of nodes) is a matter of debate in complex networks science [1-7]. Nodes failure can eliminate important connections in real systems compromising the network functioning and eventually determining the network collapse. In the last years, this topic has been intensely investigated in different fields of applied network science, such as Internet [1, 2, 8], electrical power grids [7], social [9], ecological [11-14] and 
biological $[9,14]$ networks. The network response to nodes failure is evaluated in terms of the decrease of some important network functioning indicators (e.g. the largest connected component or the network diameter) following nodes removal. The robustness of the network can then be quantified from the decrease rate of this indicator of functioning i.e. the faster is the decrease, the lower the network robustness [1-10]. The temporal sequence of node removal can be random or it can be determined by a so-called attack strategy with nodes sorted according to some topological properties [1-10].

Most of the existing analyses on the network response to nodes removal have been conducted using binary networks where the intensity (i.e. weight) of the links is not considered, and therefore the links can only be present or absent. Nonetheless, real-world networks commonly have a complex topological structure and display a large heterogeneity in the capacity and intensity of the connections [15-20] and for this reason, a comprehensive description of these systems is only possible when the heterogeneity in links weight is considered $[15,16]$.

In a pioneering work of Dall'Asta et al. [21] investigated the response of the worldwide airport weighted network to different attack strategies using binary and weighted measurements of the system functioning. They found a lower network robustness when the network functioning is evaluated with weighted, rather than with binary, measurements [21].

Wang and Chen [22] analyzed weighted network models against nodes failure and by applying the local weighted flow redistribution rule they found the strongest robustness against cascaded failure for a specific shape of the distribution of links weight [22]. Differently, Mirzasoleiman e al. [23] , rather than nodes failure, investigated the robustness of networks focusing on links removal. They analyzed a number of factors influencing the robustness of model networks against the removal of links with maximum weight where networks links were weighted according to different rules. They found that the networks whose links weight is the product of the betweenness centrality of the end nodes have the largest robustness against the failure of links of maximum weight [23]. Bellingeri 
and Cassi [24] analyzed the response to nodes failure accounting for link intensity and the weighted structure of model and real-world networks introducing a weighted rank for node importance (node strength) in the attack strategies, and used a measurement for network functioning that accounts for the weight of the links (weighted efficiency). They found that the efficiency of the attack strategies changed when passing from binary to weighted network functioning measurements; in addition, they show that if the weighted structure of complex networks is not considered, this may produce misleading models to forecast the system response to node failure, i.e. only considering binary links may not unveil the real damage induced to the system [24]. In a recent paper Bellingeri et al. [25] analyzed the response to attack strategies of the real-world weighted network of the urban taxi traffic system of Beijing using weighted and binary measurements of the network functioning. They found that to discover the best attack strategy, i.e. the attack producing the highest damage, it is important to use node rank accounting for links weight, e.g. considering the amount of taxi flows among nodes [25].

All these research studies analyzed the response of the weighted networks under nodes removal focusing on the whole system collapse; to do this they addressed the investigation removing all the nodes investigating the system response at the end of the process, i.e. when the functioning measurement reached the quasi-zero value. However, in many cases real world networks underwent local damage of one or a small ensemble of nodes only determining a decrease in the system functioning. This is the simple case of cross-roads (or streets) closing, an everyday local failure in large real-world complex road networks, causing inconveniences and delays for the travelers, that decreases the system functioning without to provoke the whole system collapse. In addition, Bellingeri et al. [5] showed that the attack strategies can change the relative efficacy with the fraction of removed nodes, indicating that to focus the whole network collapse may be misleading when a reduced number of node to remove are available. That is why it is interesting to investigate the network response focusing on few nodes failure and the consequent decrease in system functioning. 
In this paper we address this new issue analyzing the response of the real-world network of the urban taxi traffic of Beijing [26] to few nodes removal. We focus the investigations by removing a small set of nodes, i.e. 1 and 2 nodes, and measuring the decrease in the system functioning using both binary and weighted network functioning measurements: the binary efficiency and the weighted efficiency $[24,27,28]$. We sort the efficacy of different attack strategies and we test, via a Montecarlo analysis, if the network damage produced by removing nodes according to attack strategies $[1-6,8,24]$ is bigger than that obtained by random removal.

\section{Methods}

\subsection{The Beijing road complex network}

We analysed the robustness of the real world complex networks of the $2^{\text {nd }}, 3^{\text {rd }}, 4^{\text {th }}$ and $5^{\text {th }}$ ring road of Beijing city, the capital of China and the second most populous city in the world with a wide and complex traffic roads system [26]. Each ring presents different number of nodes ranging from $\mathrm{N}=144$ to $\mathrm{N}=871$. Nodes represent the road intersections and links depict the roads connecting nodes [26]. The weights of real traffic network roads (links) can be estimated e.g. by the actual length of the road, the traffic flow of the road, the average speed of the vehicles, etc. In the present work we estimated it to be equivalent to the taxi vehicle flow, that we computed as the number of taxis passing the road from February the $2^{\text {nd }}$ to $8^{\text {th }}, 2008$. For a detailed topological analyses of the Bejing urban road network see Guo and Lu [26].

\subsection{The attack strategies}

We evaluated four attack strategies based on a binary description of the network and two based on a weighted description of the network. These strategies have been extensively presented and discussed in previous works (Bellingeri et al. [4], Bellingeri and Cassi [24]). We analyzed the response of the system under 1 and 2 nodes removal from the networks.

\section{Binary attack strategies.}


- Nearest neighbors (First): nodes are sequentially removed according to the number of nearest neighbors of each node (i.e. node degree) $[8,4]$.

- Next to nearest neighbors $(\mathrm{Sec})$ : nodes are removed according to the number of nodes connected to the nearest neighbors of that node.

- Next to nearest neighbors plus nearest neighbors $(F+S)$ : nodes are removed according to the number of nearest neighbors plus nodes connected to the nearest neighbors of that node.

- Nodes betweenness centrality (Bet): nodes are sequentially removed according to their betweenness centrality, which is the number of shortest paths from all vertices to all the others that pass through that node [29].

\section{Weighted attack strategies.}

- Google Page Rank (Goog): nodes are deleted according to the rank produced by the algorithm used by Google to rank their search engine results [24,30].

- The strength attack (Str): nodes are removed according to the node strength. The node strength of a given node is the sum of the weight of the links incident to the node [21,24].

For all the strategies in the case of ties (i.e. nodes with the same property parameter), the sequence of nodes removal is randomly chosen.

\subsection{Damage characterization: the network efficiency}

We quantified the efficiency of the network during the node removal process. The efficiency of a network is based on the shortest path notion. The shortest path between two nodes is the minimum number of links needed to travel between the nodes. In a binary network, the shortest path between two nodes is an integer number indicating the minimum amount of links necessary to travel from a node to the other [31]. In a weighted network, a path between two nodes is the sum of the weights associated to the links needed to travel between the nodes [31]. The efficiency for a pair of nodes is the reciprocal of the shortest path between the nodes (both for binary or weighted network). We 
referred to binary efficiency (Bin EFF) when links weight is not considered in the measurement, and weighted efficiency (Weg EFF) when weights are included [24,28]. The higher the shortest path between two nodes, the lower the efficiency $[24,28]$. Since, the shortest path measurement of the entire network is the average of shortest paths among each couple of nodes, the efficiency of a network is the average of the efficiency between each couple of nodes. See Appendix A.1 for a detailed mathematical explanation of the network efficiency measurements.

\subsection{The Montecarlo simulations}

We assessed the uncertainty in the estimate of the attack strategies efficacy via a Montecarlo randomization test, with random removal sequences resampled 10,000 times, and confidence intervals computed with the percentile method [32]. Namely:

1) We iterated 10,000 random removal sequences for each ring roads of the Beijing road traffic complex network and we measured the networks efficiency.

2) We computed the difference in the network efficiency between the initial efficiency (e.g. the network efficiency before any nodes removal) and the network efficiency after the removal of 1 and 2 nodes. We refer to this difference as to the network damage (Damage).

3) We compare the empirical null Damage distribution obtained from points 1) and 2) with the damage produced by the selective attack strategies using confidence intervals with the percentile method. In the null hypothesis the damage produced by selective attack strategies does not differ from the empirical null Damage distribution. We count the number of Damages obtained by random removal sequences higher than the damage induced by each attack strategies. This count represents the probability to have a damage caused by a random node failure higher than the one triggered by a given attack strategy and the $p$-value for the null hypothesis; we use this probability like a significance value $p$ for the efficacy of each attack strategy.

\section{Results}


The empirical null distributions of the damages (Damage) are mostly bell shaped (See Figs 1-4). For each Damage distribution we fitted a normal distribution computing the mean $(\mu)$ and the standard deviation $(\sigma)$. The computed normal distributions parameters are reported in Table A.1 in the Appendix. The normal distribution fitting are depicted in Figs A.3-A.6 in the Appendix.

We found the attack strategies to generally be more efficient than the random removal to decrease the networks functioning. Figs 1-4 show how the selective attack strategies, with differences, produced an overall damage higher than the mean Damage caused by random node removals and in most cases, the attack strategies produced Damage higher than $95 \%$ of the random removal sequences. The percentile values representing the probability to have random removal sequences producing higher Damage than a given attack strategy in the Damage distribution ensemble are reported in Table 1.

$2^{\text {nd }}$ ring: when the damage is measured by Weg Eff, the best attack strategies are Bet and Goog for 1 and 2 removals (Fig. 1). Sec and $F+S$ are non effective with 0.52 and 0.41 random damage higher than the damage produced by these strategies. Using the Bin Eff Bet becomes the best strategy with $p=0.0001$ followed by Str and First with equal efficacy (Fig. 2); interestingly, Sec and $F+S$ become more efficient when the damage is measured by Weg Eff with $p=0.0451$ and 0.002 .

$3^{\text {rd }}$ ring: using Weg Eff Str is the best strategy for 1 removal and Goog is best for 2 removals (Fig. 2); Bet follows in third position; Sec and $F+S$ are generally less efficient and, interestingly, First produces small damage and it does not differ from the null hypothesis with $p=0.1605$ and 0.3274 for 1 and 2 removals respectively. For Bin Eff, Bet is the best strategy with no Damage distribution outcomes higher than it $(p=0)$.

$4^{\text {th }}$ ring: using Weg Eff Str is clearly the best strategy for both 1 and 2 removals (Fig. 3) with no random Damage higher than it; Bet follows in second position; Sec and $F+S$ are generally less efficient and, interestingly, First, the binary counterpart of Str, is not efficient for 1 and 2 removals 
respectively ( $p=0.28$ and 0.26). For Bin Eff, Bet is the best strategy with no Damage distribution outcomes higher than it $(p=0)$.

$5^{\text {th }}$ ring: like the $4^{\text {th }}$ ring, using Weg Eff Str is clearly the best strategy for both 1 and 2 removals (Fig. 4) with no random Damage higher than it ( $p=0)$; Goog results in second position; $S e c$ and $F+S$ are generally less efficient. It is worth nothing that using Weg Eff, First and Bet are not efficient for 1 removal and they not differ from null hypothesis with $p=0.441$ for both strategies, but they become efficient removing 2 nodes with $p=0$ for both strategies. For Bin Eff Bet results the most efficient strategy for both 1 and 2 removals with $p=0$. Interestingly, Sec resulting no efficient when the damage is measured by the Weg Eff, is now the second strategy with $p=0.0143$ and 0 , followed by $F+S$. The weighted strategies $S t r$ and Goog produce the lowest damage.

\section{Discussion}

In this paper we addressed the general problem of real-world network robustness focusing on the system reaction to few local node failures and comparing the outcomes with classical analyses investigating the whole system collapse (cascade of failures). To do this, we analyzed the response to 1 and 2 node removals in terms of robustness of the real world weighted complex networks of the $2^{\text {nd }}, 3^{\text {rd }}, 4^{\text {th }}, 5^{\text {th }}$ ring roads of Beijing city using weighted and binary measurements of the damage and weighted and binary properties to select the nodes to remove. We found that, in 3 out of 4 rings, the weighted node attack strategy Str that remove nodes according to their strength, i.e. the sum of the weight of the links to the node, is more efficient in producing damages when we measured the network functioning with the weighted efficiency (Figs 1-4 and Table 1). In other terms, Str is the most powerful strategy to individuate important nodes in the analyzed network. Since in our network the weight of the links indicates the amount of taxi passing along the road [26], to individuate the most important nodes in the real world road complex networks of Beijing city it is 
necessary to consider the total traffic passing in the crossroads (node) and not only the binary topological structure of the roads network. This last result partially disagrees with previous analyses on the Beijing city road system producing the whole network collapse [25]. Bellingeri et al. [25] found Str to be the most efficient strategy using Weg Eff only on the $2^{\text {nd }}$ ring and for the $3^{\text {rd }}$ ring up to reach $1 / 3$ of the node removals, after this value Goog became more efficient. This difference in efficacy indicates how a local strategy like Str may be highly efficient for a few node removals, as we found here removing 1 and 2 nodes. However, when the number of node removals overcomes a given value, Goog strategy, which is able to consider the general importance of the component in the system and can be viewed as a global strategy [30], shows higher efficacy in selecting important nodes [25].

Bellingeri et al. [5] found for binary models and real-world networks, that the rank of efficacy of the removal strategies can change depending on the amount of removed nodes, indicating how commonly global used measure of the strategy efficacy considering the whole network collapse [13,9], may be misleading when just a few "shots" are available. The transition pattern in the strategies efficacy that we show here for weighted networks, where for few removals the best strategy is Str and after become Goog [25], is in agreement with the results presented for binary attack strategies in Bellingeri et al. [5]. Both works outline the importance to consider the amount of removals in order to understand the system response to different kind of node loss.

Str strategy is not the most efficient attack using the binary efficiency: i.e. when Bin Eff evaluates the network damage, Bet strategy may be more powerful to select important nodes for networks functioning (Figs 1-4 and Table 1). The higher efficacy of the Bet strategy we found here is in agreement with previous analyses showing how removing nodes according to their betweenness centrality is highly disruptive for the network functioning even when the functioning is measured by the largest connected cluster $[1,5,6]$. 
Dall'Asta et al. [21], analysing weighted airport networks, discovered that introducing weighted measurement of the damage increases the vulnerability of the network to node loss. They emphasize how the purely binary measure of the size of the largest component does not convey all the information needed [21]. Our findings are in agreement with the pioneering analyses of Dall'Asta et al. [21] showing how to adopt binary or weighted measurement in the damage analyses may clearly change the networks response to node removal. In order to discover the best attack strategies, and thus the best way to select important vertexes, it is important to analyze the network response to node loss using attack strategies accounting the magnitude of the links to the nodes, such as the nodes strength Str. In Fig. 5 we depict a simple example showing the necessity to account the weight of the links to discover important nodes. This confirms our previously analyses showing that neglecting the weighted structure of complex networks may produce misleading models to forecast the system response to node failure, i.e. not account the intensity of the links with adapt weighted measures may not unveil the real damage induced in the system $[29,24,25,33]$. A similar pattern is founded in both physics [24,25] and ecological networks, such as food webs [19,33].

Guo and Lu [26] analyzed Beijing road networks used in this study and found that the maximum nodes degree and the degree distributions are the same throughout rings hence the number of nodes and the diameter sharply increase from $2^{\text {nd }}$ to $5^{\text {th }}$ ring. Since $S t r$ strategy is very efficient for all the rings of the Beijing road networks (Table 1), we can argue that the efficacy of the Str strategy is also size invariant, i.e. is not affected by the number of nodes or by the paths length of the networks. Further, this last result would suggest how simple local weighted attack strategy would be adequately effective to individuate important nodes in road networks, even when a detailed global information on the network structure is not available (for example, the paths length or diameter). Given that real-world complex networks may show structure differences, it would be very interesting to test while local weighted strategy Str (or similar) is highly effective and 'sizeinvariant', like we found in our analyses, in complex systems from other fields, such as for example biological, immunological or social. This would produce implications in concrete policies, like for 
example to individuate important component supporting biological life or preferential individuals to immunize.

Testing the efficiency of the different attack strategies is extremely important because identifies the most important nodes for network functioning, and therefore which nodes should be primarily protected, as in the case of computer [1,3] or ecological networks [10,12,19,34], or removed, as in the case of immunization/disease networks [5,35]. Here we found that, even removing 1 or 2 nodes, the attack strategies are generally more efficient than the random removals; this result is in agreement with the wide literature in the field where the system response to node loss was measured removing all the nodes [1-6]. This last result would align the efficacy of our local attack analyses presented in this paper with the efficacy of the global attacks widely investigated in the literature. Nonetheless, we found how a certain fraction of damage caused by random node removals may be higher than the damage produced by the attack strategies, both for binary and weighted efficiency (Table 1). For example, for Weg Eff in the $4^{\text {th }}$ ring network all the strategies except the best strategy Str and then Bet are useless. Further, for 1 removal and both the efficiency measurements, in the $5^{\text {th }}$ ring network no one selective attack strategy presents $p=0$, i.e. no strategy performed better than all the Montecarlo outcomes. This may indicate that to find the best attack, the simulation of a higher number of random nodes removal may be necessary. We choose to analyze for the first time the network response to a small number of nodes removals because we are convinced that in real world networks it is a failure pattern with higher chance. Since the analyses presented here focused on few removals, this strategy can be available even for medium and large networks, whereas simulating a very large number of the entire removal process involving all the nodes may become quickly time consuming. For this reason, we showed how to simulate the highest number of a small number of nodes failure may be the best approach to select important nodes.

\section{Acknowledgment}


Many thanks to Stefano Poletti for useful suggestions on the final version of the paper. We thank

Giulia Leni for fruitful exchanges on the manuscript first idea.

\section{References}

[1] R. Albert, A. Barabási, Statistical mechanics of complex networks, Rev. Mod. Phys. 74 (2002).

[2] D.S. Callaway, M.E. Newman, S.H. Strogatz, D.J. Watts, Network robustness and fragility: percolation on random graphs., Phys. Rev. Lett. 85 (2000) 5468-71.

[3] R. Cohen, K. Erez, D. ben-Avraham, S. Havlin, Breakdown of the Internet under Intentional Attack, Phys. Rev. Lett. 86 (2001) 3682-3685. doi:10.1103/PhysRevLett.86.3682.

[4] M. Bellingeri, D. Cassi, S. Vincenzi, Efficiency of attack strategies on complex model and real-world networks, Phys. A Stat. Mech. Its Appl. 414 (2014) 174-180. doi:10.1016/j.physa.2014.06.079.

[5] M. Bellingeri, E. Agliari, D. Cassi, Optimization strategies with resource scarcity: from immunization of networks to the traveling salesman problem, Mod. Phys. Lett. B. (2015).

[6] T. Nie, Z. Guo, K. Zhao, Z.M. Lu, New attack strategies for complex networks, Phys. A Stat. Mech. Its Appl. 424 (2015) 248-253. doi:10.1016/j.physa.2015.01.004.

[7] L.L. Hao Yao-hui, Han Ji-hong, Yi Lin, Vulnerability of complex networks under three-level-tree attacks, Phys. A Stat. Mech. Its Appl. 462 (2015) 674-683.

[8] P. Holme, B.J. Kim, C.N. Yoon, S.K. Han, Attack vulnerability of complex networks, Phys. Rev. E. 65 (2002) 56109.

[9] S. Iyer, T. Killingback, B. Sundaram, Z. Wang, Attack robustness and centrality of complex networks., PLoS One. 8 (2013) e59613. doi:10.1371/journal.pone.0059613.

[10] A. Bodini, M. Bellingeri, S. Allesina, C. Bondavalli, Using food web dominator trees to catch secondary extinctions in action., Philos. Trans. R. Soc. Lond. B. Biol. Sci. 364 (2009) 1725-31. doi:10.1098/rstb.2008.0278. 
[11] M. Bellingeri, A. Bodini, Threshold extinction in food webs, Theor. Ecol. 6 (2013) 143-152.

[12] M. Bellingeri, D. Cassi, S. Vincenzi, Increasing the extinction risk of highly connected species causes a sharp robust-to-fragile transition in empirical food webs, Ecol. Modell. 251 (2013) 1-8.

[13] E. Calizza, M.L. Costantini, L. Rossi, Effect of multiple disturbances on food web vulnerability to biodiversity loss in detritus-based systems, Ecosphere. 6 (2015) 1-20. doi:10.1890/ES14-00489.1.

[14] H. Jeong, R. Albert, Z.N. Ottval, A.L. Barabási, The large scale organization of metabolic networks, Nature. 407 (2000) 651-654.

[15] A. Allard, M.Á. Serrano, G. García-Pérez, M. Boguñá, The geometric nature of weights in real complex networks, Nat. Commun. 8 (2017) 1-8. doi:10.1038/ncomms14103.

[16] D. Garlaschelli, The weighted random graph model, New J. Phys. 11 (2009) 73005. doi:10.1088/1367-2630/11/7/073005.

[17] A. Barrat, M. Barthélemy, R. Pastor-Satorras, A. Vespignani, The architecture of complex weighted networks., Proc. Natl. Acad. Sci. U. S. A. 101 (2004) 3747-3752. doi:10.1073/pnas.0400087101.

[18] M. Barthélemy, A. Barrat, R. Pastor-Satorras, A. Vespignani, Characterization and modeling of weighted networks, Phys. A Stat. Mech. Its Appl. 346 (2005) 34-43. doi:10.1016/j.physa.2004.08.047.

[19] M. Bellingeri, A. Bodini, Food web's backbones andenergy delivery in ecosystems, Oikos. (2015).

[20] E. Agliari, a. Barra, A Hebbian approach to complex-network generation, EPL (Europhysics Lett. 94 (2011) 10002. doi:10.1209/0295-5075/94/10002.

[21] L. Dall'Asta, A. Barrat, M. Barthélemy, A. Vespignani, Vulnerability of weighted networks, J. Stat. Mech. Theory Exp. 4006 (2006). doi:10.1088/1742-5468/2006/04/P04006.

[22] W.X. Wang, G. Chen, Universal robustness characteristic of weighted networks against cascading failure, Phys. Rev. E - Stat. Nonlinear, Soft Matter Phys. 77 (2008) 1-5. doi:10.1103/PhysRevE.77.026101. 
[23] B. Mirzasoleiman, M. Babaei, M. Jalili, M. Safari, Cascaded Failure in Weighted Networks, Phys. Rev. E. 84 (2011) 46114. doi:10.1103/PhysRevE.00.006100.

[24] M. Bellingeri, D. Cassi, Robustness of weighted networks, Phys. A Stat. Mech. Its Appl. 489 (2018) 47-55. doi:10.1016/j.physa.2017.07.020.

[25] M. Bellingeri, Z.M. Lu, D. Cassi, F. Scotognella, Analyses of the response of a complex weighted network to nodes removal strategies considering links weight: the case of the Bejing urban road system, Mod. Phys. Lett. B. (2018) In publication.

[26] X. Guo, Z.-M. Lu, Urban Road Network and Taxi Network Modeling Based on Complex Network Theory, J. Inf. Hiding Multimed. Signal Process. 7 (2016) 558-568.

[27] P. Crucitti, V. Latora, M. Marchiori, A. Rapisarda, Error and attack tolerance of complex networks, Phys. A Stat. Mech. Its Appl. 340 (2004) 388-394. doi:10.1016/j.physa.2004.04.031.

[28] V. Latora, M. Marchiori, Efficient Behavior of Small-World Networks, Phys. Rev. Lett. 87 (2001) 198701. doi:10.1103/PhysRevLett.87.198701.

[29] M. Barthélemy, Betweenness centrality in large complex networks, Eur. Phys. J. B. 38 (2004) 163168.

[30] S. Brin, L. Page, The Anatomy of a Large-Scale Hypertextual Web Search Engine, in: Seventh Int. World-Wide Web Conf., 1998.

[31] D.B. West, Introduction to Graph Theory., Prentice Hall., 1996.

[32] G. Nicholas, A. Ellison, A primer of ecological statistics, Sinauer, 2004.

[33] M. Bellingeri, S. Vincenzi, Robustness of empirical food webs with varying consumer's sensitivities to loss of resources, J. Theor. Biol. (2013).

[34] R. V Solé, J.M. Montoya, Complexity and fragility in ecological networks., Proc. Biol. Sci. 268 (2001) 2039-45. doi:10.1098/rspb.2001.1767. 
[35] R. Pastor-Satorras, A. Vespignani, Immunization of complex networks, Phys. Rev. E. 65 (2002) 36104. doi:10.1103/PhysRevE.65.036104.

\section{Figures}

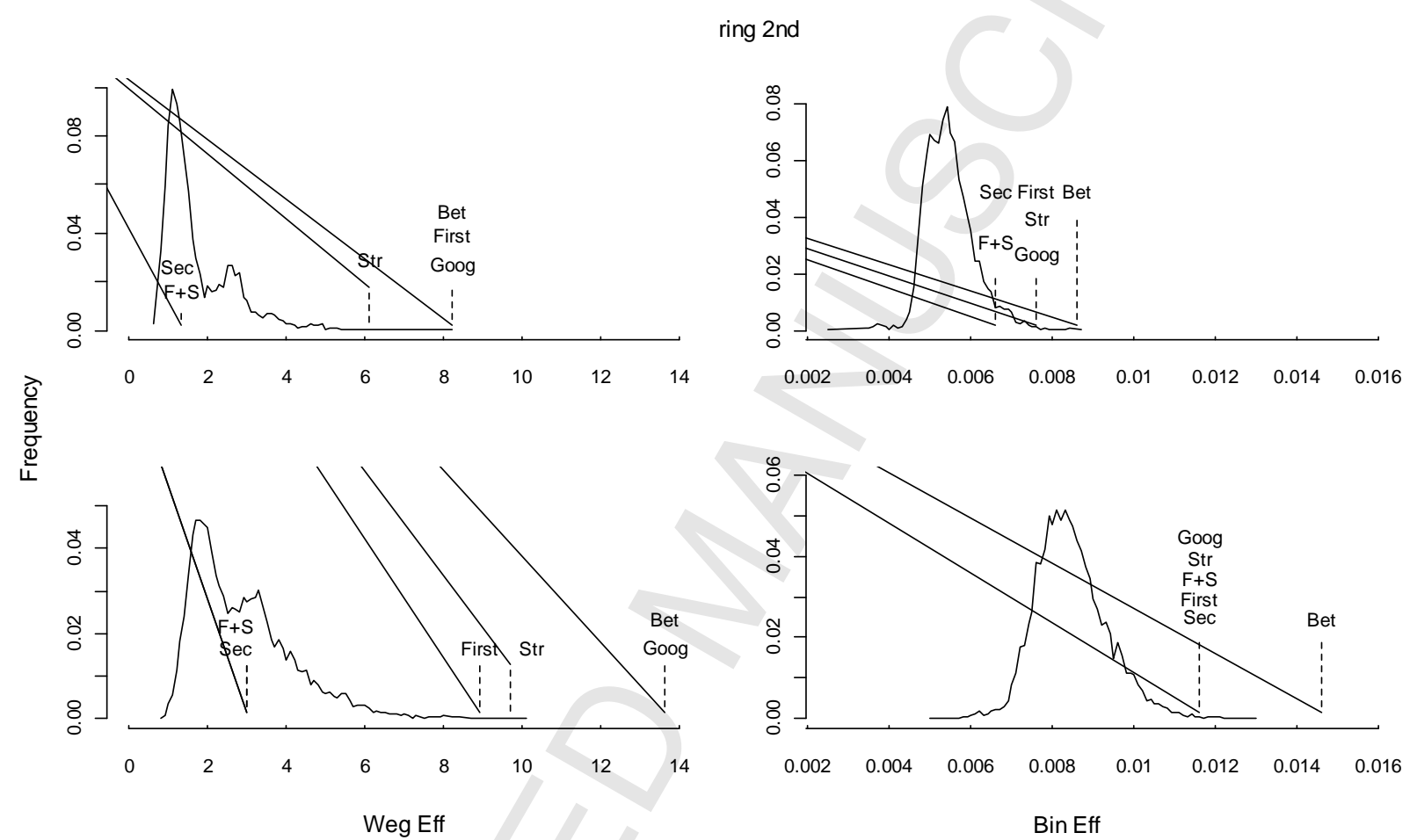

Figure 1: Damage distributions for the $2^{\text {nd }}$ ring of the Beijing road traffic networks for weighted and binary efficiency; top row 1 node removal, bottom row 2 node removals. 
ring 3rd
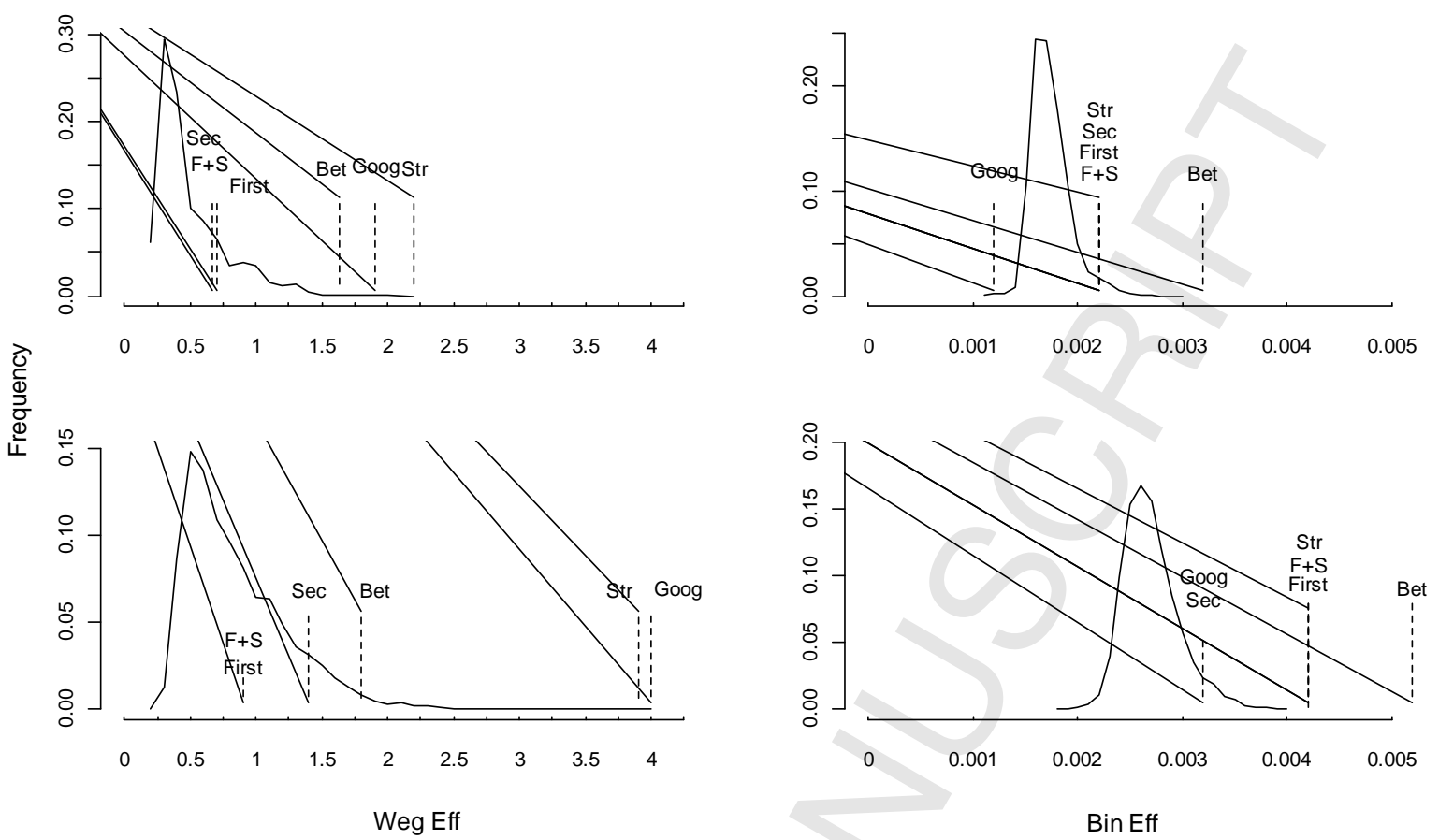

Figure 2: Damage distributions for the $3^{\text {rd }}$ ring of the Beijing road traffic networks for weighted and binary efficiency; top row 1 node removal, bottom row 2 node removals.

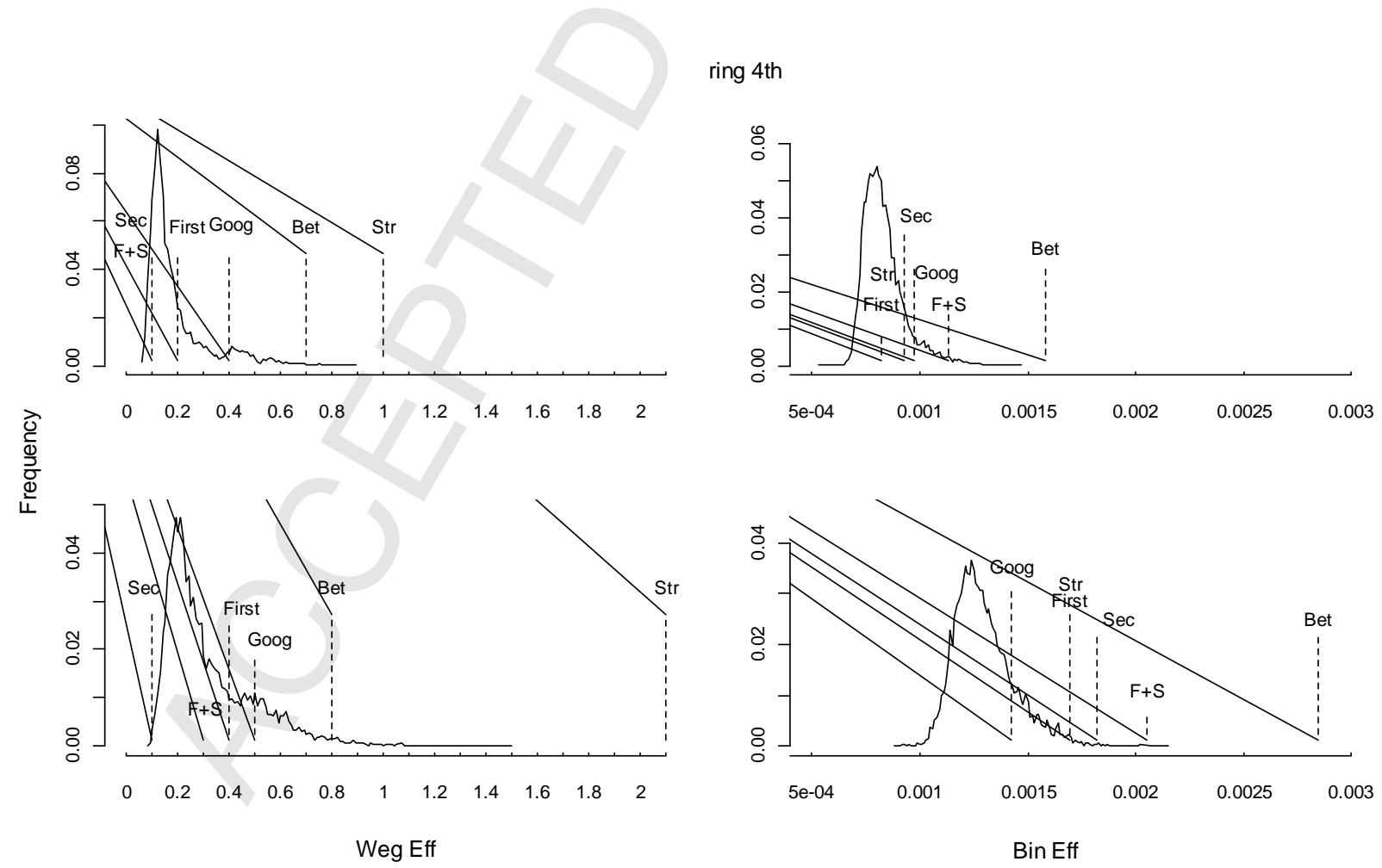


Figure 3: Damage distributions for the $4^{\text {th }}$ ring of the Beijing road traffic networks for weighted and binary efficiency; top row 1 node removal, bottom row 2 node removals.

ring 4 th
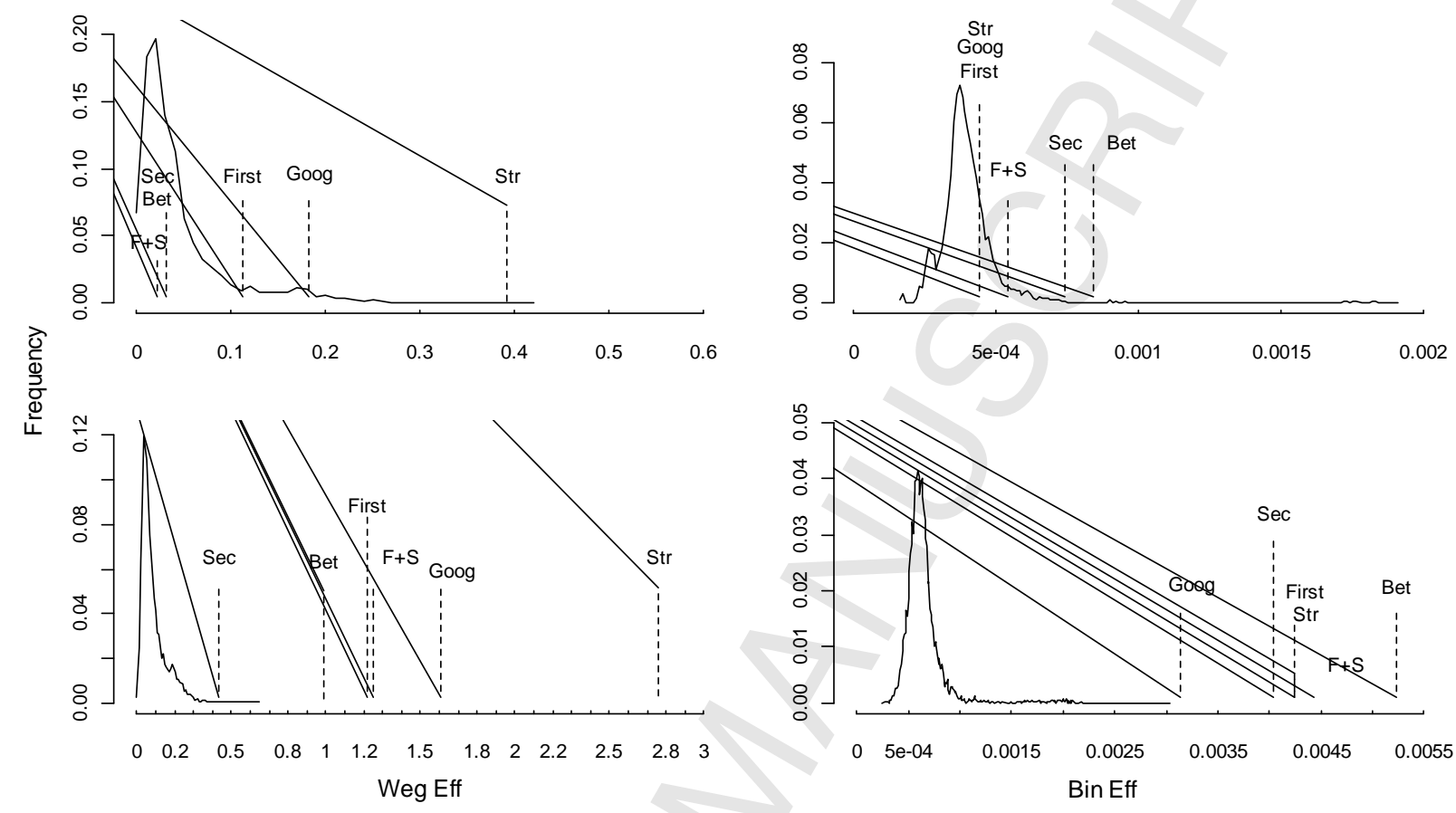

Figure 4: Damage distributions for the $5^{\text {th }}$ ring of the Beijing road traffic networks for weighted and binary efficiency; top row 1 node removal, bottom row 2 node removals. 

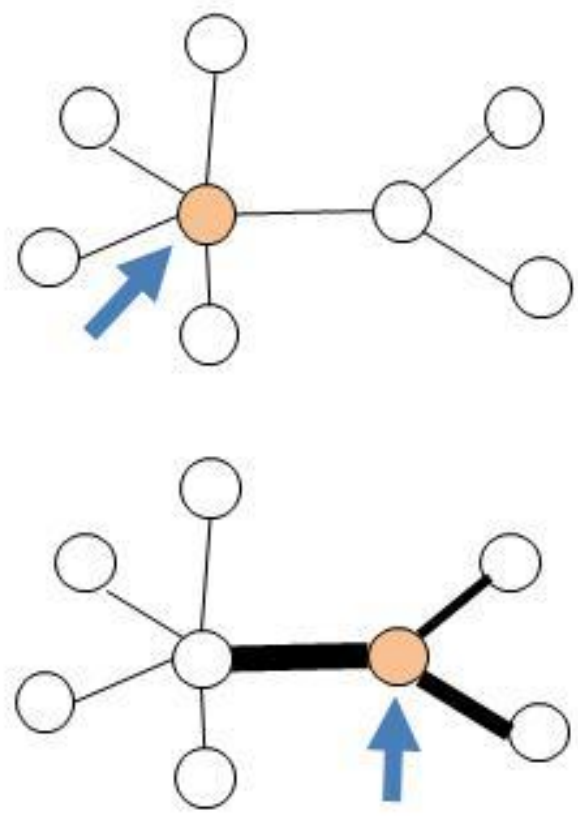

Figure 5: Simple example showing that neglecting the weighted structure of complex networks may produce misleading models to forecast the system response to node failure. Binary (top figure) and weighted (bottom) models of the same complex network. The failure of the nodes with higher binary degree (top) may not damage the most important node when the weight of the links are considered in the network (bottom).

Table 1: fraction of random removal producing a damage higher than the selective attack strategies.

\begin{tabular}{|l|l|l|l|l|}
\hline \multicolumn{5}{|l|}{$2^{\text {nd }}$} \\
\hline & Weighted & \multicolumn{2}{l|}{} \\
\hline & 1 Shot & 2 Shots & 1 Shot & 2 Shots \\
\hline First & 0 & 0.0007 & 0.0026 & 0.002 \\
\hline Sec & 0.5275 & 0.4135 & 0.0451 & 0.002 \\
\hline F+S & 0.5275 & 0.4135 & 0.0451 & 0.002 \\
\hline Goog & 0 & 0 & 0.0026 & 0.002 \\
\hline
\end{tabular}




\begin{tabular}{|c|c|c|c|c|}
\hline Bet & 0 & 0 & 0.0001 & 0 \\
\hline Str & 0.0011 & 0.0002 & 0.0026 & 0.002 \\
\hline \multicolumn{5}{|c|}{$3^{\text {rd }}$} \\
\hline & \multicolumn{2}{|c|}{ Weighted } & \multicolumn{2}{|l|}{ Binary } \\
\hline & 1 Shot & 2 Shots & 1 Shot & 2 Shots \\
\hline First & 0.1605 & 0.3274 & 0.0218 & 0 \\
\hline $\mathrm{Sec}$ & 0.2256 & 0.114 & 0.0218 & 0.0428 \\
\hline$F+S$ & 0.1605 & 0.3274 & 0.0218 & 0 \\
\hline Goog & 0.0003 & 0 & 0.996 & 0.0428 \\
\hline Bet & 0.0028 & 0.0263 & 0 & 0 \\
\hline Str & 0 & 0.0001 & 0.0218 & 0 \\
\hline \multicolumn{5}{|c|}{$4^{\text {th }}$} \\
\hline & \multicolumn{2}{|c|}{ Weighted } & \multicolumn{2}{|l|}{ Binary } \\
\hline & 1 Shot & 2 Shots & 1 Shot & 2 Shots \\
\hline First & 0.2799 & 0.256 & 0.6261 & 0.0122 \\
\hline $\mathrm{Sec}$ & 0.8841 & 0.9984 & 0.2128 & 0.0037 \\
\hline$F+S$ & 0.8841 & 0.406 & 0.0274 & 0.0001 \\
\hline Goog & 0.0943 & 0.1607 & 0.0747 & 0.2165 \\
\hline Bet & 0.0044 & 0.0186 & 0 & 0 \\
\hline \multirow[t]{4}{*}{ Str } & 0 & 0 & 0.6261 & 0.0122 \\
\hline & \multicolumn{3}{|c|}{$5^{\text {th }}$} & \\
\hline & \multicolumn{2}{|c|}{ Weighted } & \multicolumn{2}{|l|}{ Binary } \\
\hline & 1 Shot & 2 Shots & 1 Shot & 2 Shots \\
\hline First & 0.0929 & 0 & 0.2056 & 0 \\
\hline $\mathrm{Sec}$ & 0.441 & 0.001 & 0.0143 & 0 \\
\hline$F+S$ & 0.6088 & 0 & 0.0606 & 0 \\
\hline Goog & 0.0297 & 0 & 0.2056 & 0 \\
\hline
\end{tabular}




\begin{tabular}{|l|l|l|l|l|}
\hline Bet & 0.441 & 0 & 0.0129 & 0 \\
\hline Str & 0.0001 & 0 & 0.2056 & 0 \\
\hline
\end{tabular}

\section{Appendix A.}

\section{A.1. The efficiency (EFF)}

We measured the functioning of the network during the nodes removal process using binary (Bin Eff) and weighted efficiency (Weg Eff) as a measure of the network efficiency [24]. The efficiency measurement is based on the shortest paths notion.

Let be $\mathrm{G}$ a binary network of $N$ nodes and $L$ links, it can be represents by an $N \times N$ binary adjacency matrix A, where the element $a_{i j}=1$ if there is a link between nodes $i$ and $j$ and 0 otherwise. A weighted network $\mathrm{G}_{\mathrm{w}}$ can be represents by a $N \times N$ matrix $\mathrm{W}$ where elements $w_{i j}>0$ if there is a links of weight $w$ between nodes $i$ and $j$. In the binary network $\mathrm{G}$, the shortest path between two nodes is an integer number indicating the minimum amount of links necessary to travel from a node to the other. In the weighted network $G_{w}$, a path between two nodes is the sum of the weight associated to the links necessary to travel between the nodes; consequently, the weighted shortest path is the minimum sum of the weights necessary to travel between the nodes [31]. Thus, the shortest path between nodes $i$ and $j$ is the minimum number of links (binary or weighted) necessary to travel between the nodes; let $p_{i j}$ the ensemble of all paths between nodes $i$ and $j$, the shortest path $s_{i j}$ of a network is:

$s_{i j}=\min \left\{p_{i j}\right\}(1)$ 
where $0<s_{i j}<\infty$ if there is a path between nodes $i$ and $j$, and $s_{i j}=\infty$ otherwise (e.g. nodes belong to different isolated clusters). The shortest path measure of a networks is the average of the minimum number of links necessary to travel among each couple of nodes [31].

We can compute a $N \times N$ efficiency matrix E where the elements $e_{i j}$ is:

$e_{i j}=\frac{1}{s_{i j}}(2)$

where $e_{i j}=0$ in the case there is no path between nodes $i$ and $j$, and $0<s_{i j}<\frac{1}{N-1}$ otherwise.

Averaging the efficiency among nodes, we can define the network efficiency EFF:

$$
E F F=\frac{1}{N(N-1)} \sum_{N}^{i, j=1} e_{i j}
$$

The efficiency computed in Eq. (3) rules for binary (Bin Eff) and weighted networks (Weg Eff).

See Figure A.1 for example of the binary and weighted networks and the associated shortest paths.

In order to assign more importance to the bigger links, we compute the reciprocal of the link weight.

To compute the shortest paths, we:

i) Compute the reciprocal of each link weight. This way, links with higher weight value represent "wider and faster routes" or, in terms of distance, "shorter routes", producing lower shortest paths. See Fig. A.2 (a) for a representation of the weighted network in Fig. A.1 (b).

ii) Compute the shortest paths for each node pairs (Eq. 1). See Figure A.2 (b). 
iii) Compute the efficiency for each pair of nodes by producing the reciprocal of the shortest paths

(Eq. 2) (See Fig. A.2 (c)). The higher the shortest paths between two nodes, the lower the efficiency.

iv) We sum the efficiency values and we average on the total number of node pairs (i.e. $N(N-1) / 2$ ).

The average value of the nodes efficiency is the network weighted efficiency (EFF) (Eq. 3).

Computing the reciprocal means that shorter routes among nodes increase the efficiency of the network and viceversa. Further, in the case two nodes are not joined by paths (as in the case of isolated clusters), the shortest path $s_{i j}$ return the infinite value for the nodes pairs. Thus, computing the reciprocal of the shortest path $s_{i j}$ for a disjoined couple of nodes $i$ and $j$ equals zero (lowest efficiency), maintaining the general idea about network efficiency.

(a)

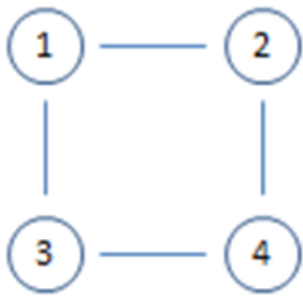

(c)

\begin{tabular}{|l|l|l|l|l|l|}
\hline & 1 & 2 & 3 & 4 & 5 \\
\hline 1 & 0 & 1 & 1 & 2 & Inf \\
\hline 2 & 1 & 0 & 2 & 1 & Inf \\
\hline 3 & 1 & 2 & 0 & 1 & Inf \\
\hline 4 & 2 & 1 & 1 & 0 & Inf \\
\hline 5 & Inf & Inf & Inf & Inf & Inf \\
\hline
\end{tabular}

(b)

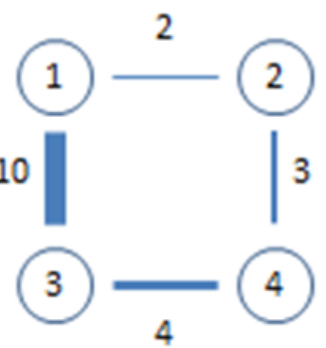

(d)

\begin{tabular}{|l|l|l|l|l|l|}
\hline & 1 & 2 & 3 & 4 & 5 \\
\hline 1 & 0 & 2 & 10 & 5 & Inf \\
\hline 2 & 2 & 0 & 7 & 3 & Inf \\
\hline 3 & 10 & 7 & 0 & 4 & Inf \\
\hline 4 & 5 & 3 & 4 & 0 & Inf \\
\hline 5 & Inf & Inf & Inf & Inf & Inf \\
\hline
\end{tabular}


Figure A.1: (a) $N \times N$ adjacency matrix of the binary network $\mathrm{G}$ with no weights associated to the links; (b) $N \times N$ matrix $\mathrm{W}$ of the weighted networks $\mathrm{G}_{\mathrm{w}} \mathrm{W}$ with links weight; (c) $N \times N$ matrix $\mathrm{S}$ of the shortest paths of the binary network in (a); $N \times N$ matrix $\mathrm{S}$ of the shortest paths of the weighted network in (b).

(a)

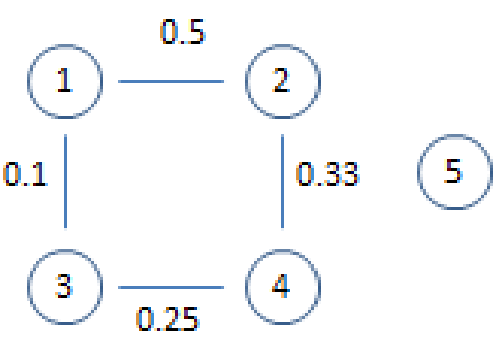

(c)

\begin{tabular}{|l|l|l|l|l|l|}
\hline & 1 & 2 & 3 & 4 & 5 \\
\hline 1 & 0 & 2 & 10 & 2.87 & 0 \\
\hline 2 & 2 & 0 & 1.7 & 3 & 0 \\
\hline 3 & 10 & 1.7 & 0 & 4 & 0 \\
\hline 4 & 2.86 & 3 & 4 & 0 & 0 \\
\hline 5 & 0 & 0 & 0 & 0 & 0 \\
\hline
\end{tabular}

(b)

\begin{tabular}{|l|l|l|l|l|l|}
\hline & 1 & 2 & 3 & 4 & 5 \\
\hline 1 & 0 & 0.5 & 0.1 & 0.35 & Inf \\
\hline 2 & 0.5 & 0 & 0.58 & 0.33 & Inf \\
\hline 3 & 0.1 & 0.58 & 0 & 0.25 & Inf \\
\hline 4 & 0.35 & 0.33 & 0.25 & 0 & Inf \\
\hline 5 & Inf & Inf & Inf & Inf & Inf \\
\hline
\end{tabular}

Figure A.2: (a) network $(N=5, L=4)$ of Fig. 2 with the reciprocal of the links weight; (b) $N \times N$ matrix of the weighted shortest paths computed for every pairs of nodes; (c) $N \times N$ matrix of the efficiencies $e_{i j}$ computed for each pairs of nodes.

\section{A.2. The Damage distributions}



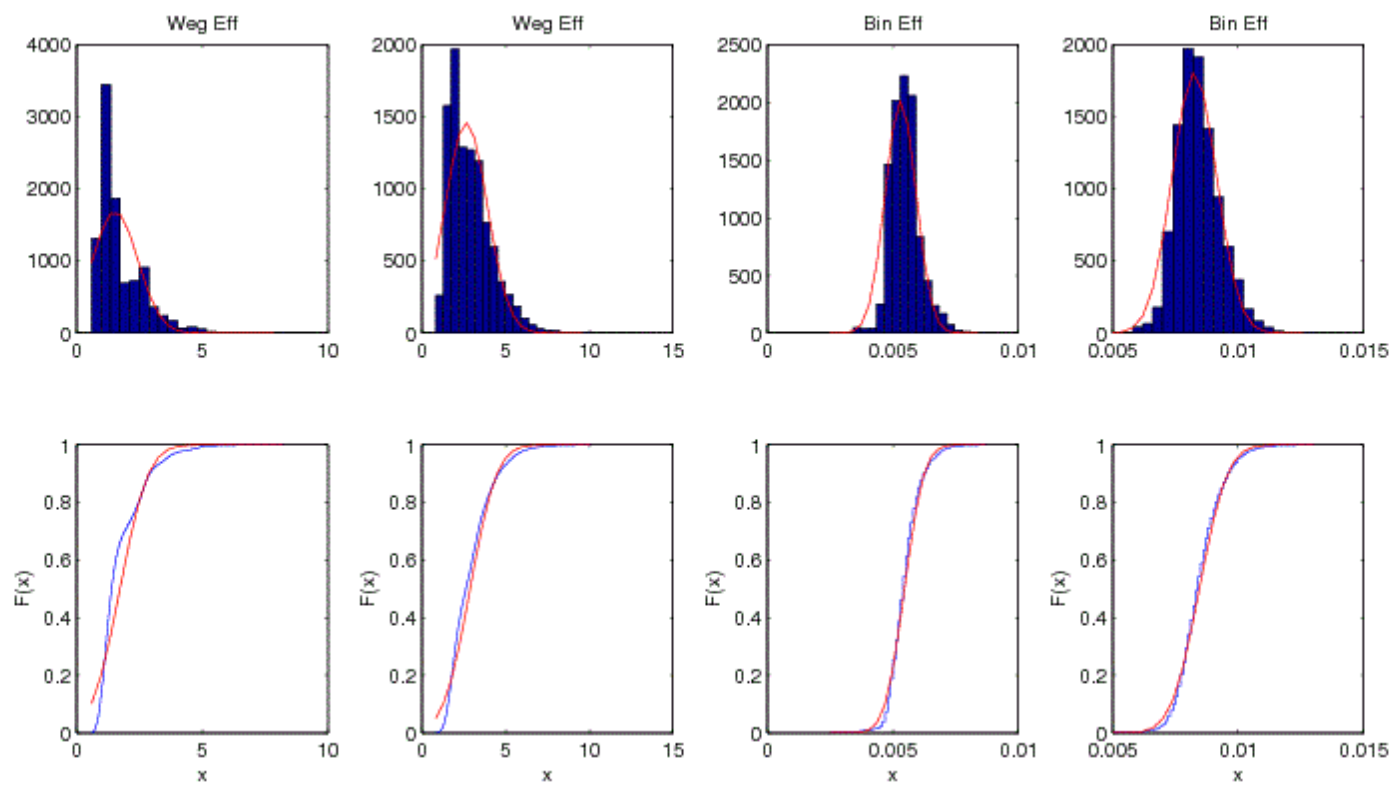

Figure A.3: Damage distributions for the $2^{\text {nd }}$ ring of the Beijing road traffic networks for weighted and binary efficiency; left column 1 node removal, right column 2 node removals. Top row describes the normal distributions fitting with the parameters $\mu$ and $\sigma$ in Table A.1; blue histograms: experimental data, red lines: normal distribution function fitting. Bottom row depict the cumulative normal distributions. 

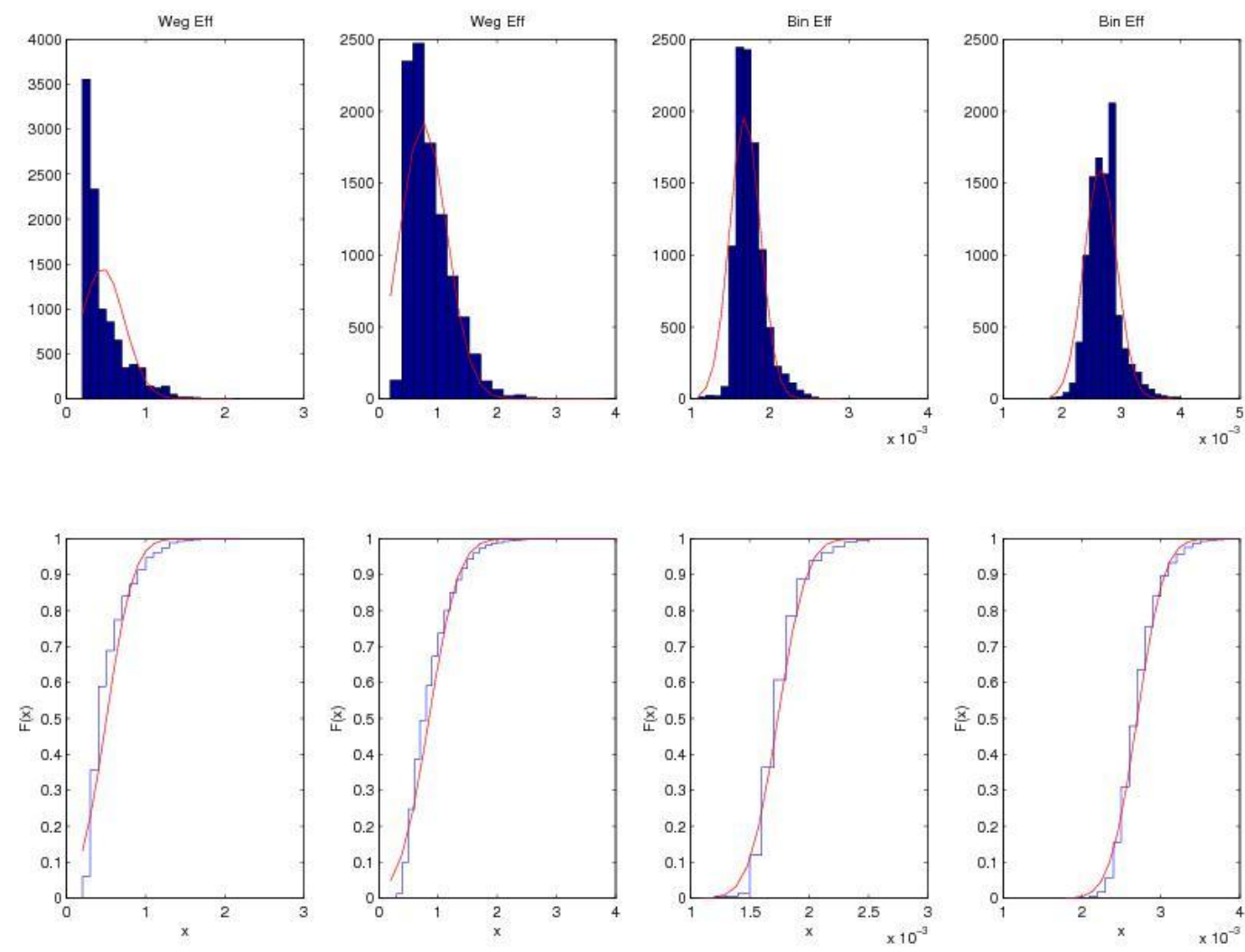

Figure A.4: Damage distributions for the $3^{\text {rd }}$ ring of the Beijing road traffic networks for weighted and binary efficiency; left column 1 node removal, right column 2 node removals. Top row describes the normal distributions fitting with the parameters $\mu$ and $\sigma$ in Table A.1; blue histograms: experimental data, red lines: normal distribution function fitting. Bottom row depict the cumulative normal distributions. 

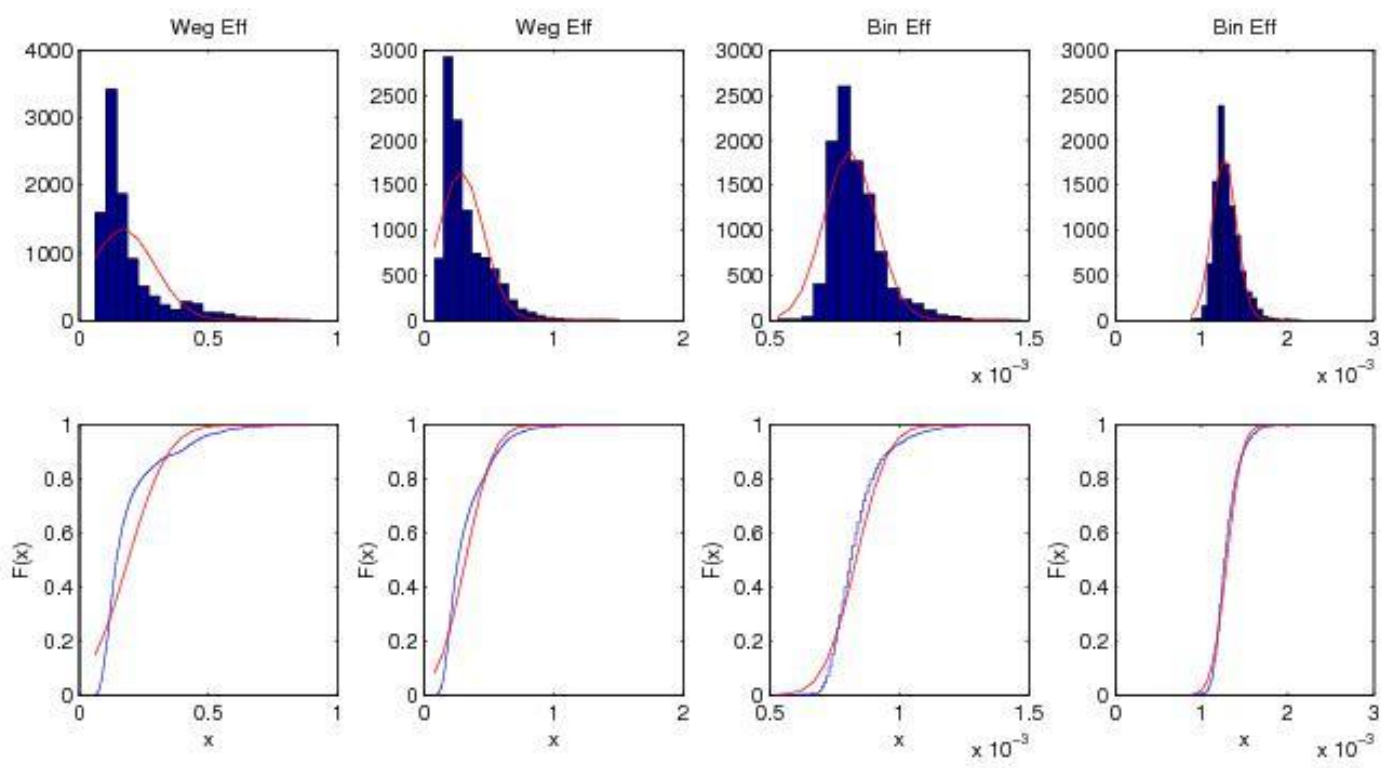

Figure A.5: Damage distributions for the $4^{\text {th }}$ ring of the Beijing road traffic networks for weighted and binary efficiency; left column 1 node removal, right column 2 node removals. Top row describes the normal distributions fitting with the parameters $\mu$ and $\sigma$ in Table A.1; blue histograms: experimental data, red lines: normal distribution function fitting. Bottom row depict the cumulative normal distributions.
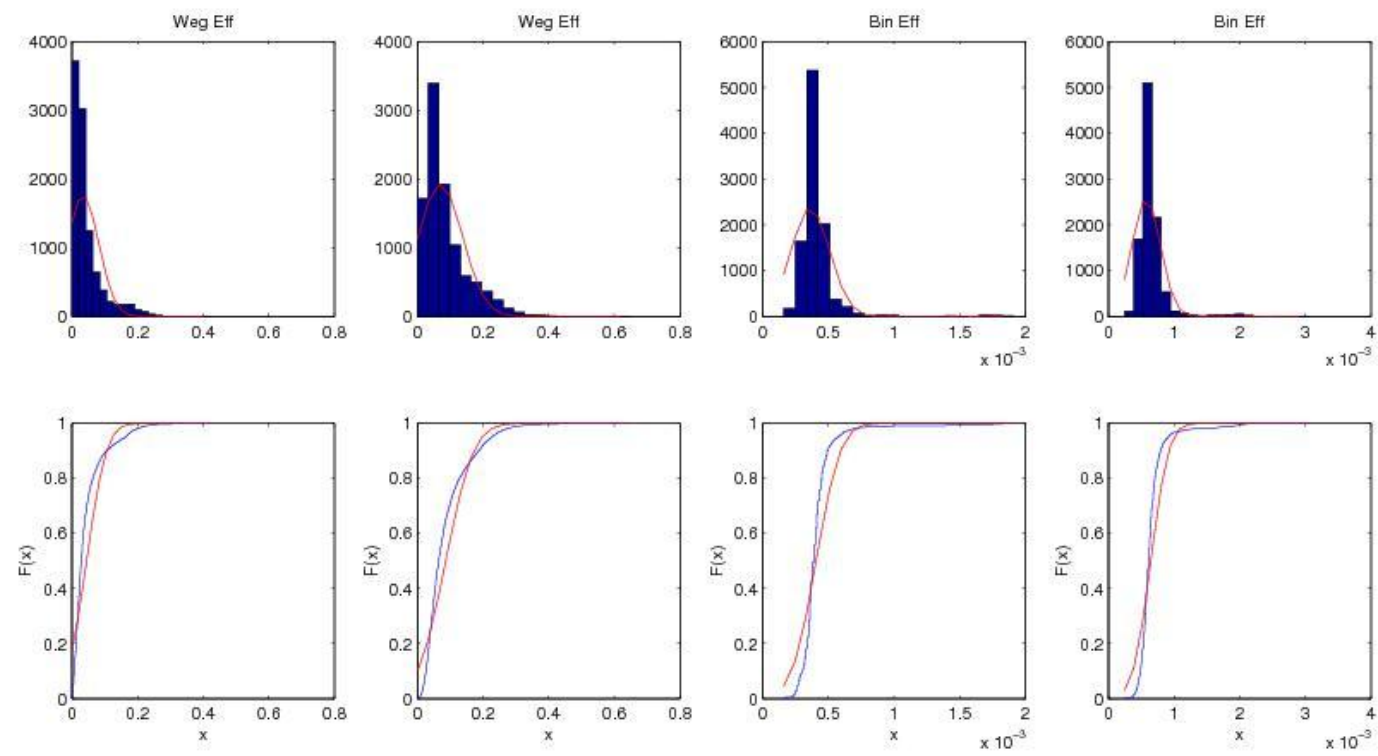

Figure A.6: Damage distributions for the $5^{\text {th }}$ ring of the Beijing road traffic networks for weighted and binary efficiency; left column 1 node removal, right column 2 node removals. Top row 
describes the normal distributions fitting with the parameters $\mu$ and $\sigma$ in Table A.1; blue histograms: experimental data, red lines: normal distribution function fitting. Bottom row depict the cumulative normal distributions.

Table A.1: Normal distributions parameters $\mu$ and $\sigma$ of the networks Damage.

\begin{tabular}{|c|c|c|c|c|}
\hline & & $2^{\text {nd }}$ ring & 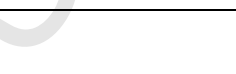 & \\
\hline & & Eff & & Eff \\
\hline & 1 removal & 2 removals & 1 removal & 2 removals \\
\hline$\mu$ & 1.72278 & 2.87381 & 0.00546438 & 0.00845544 \\
\hline$\sigma$ & 0.890324 & 1.25396 & 0.000607007 & 0.000874394 \\
\hline & & $3^{\text {rd }}$ ring & & \\
\hline & & Eff & & Eff \\
\hline & 1 remova & 2 removals & 1 removal & 2 removals \\
\hline$\mu$ & 0.5049 & 0.850030 & 0.00173488 & 0.00270 \\
\hline$\sigma$ & 0.272821 & 0.3910336 & 0.0001911 & 0.000272 \\
\hline & & $4^{\text {th }}$ ring & & \\
\hline & & Eff & & Eff \\
\hline & 1 removal & 2 removals & 1 removal & 2 removals \\
\hline$\mu$ & 0.1895849 & 0.31856 & 0.00083359 & 0.0012970 \\
\hline$\sigma$ & 0.122878 & 0.172182 & 0.000088899 & 0.00013995 \\
\hline & & $5^{\text {th }}$ ring & & \\
\hline
\end{tabular}


ACCEPTED MANUSCRIPI

\begin{tabular}{|l|l|l|l|l|}
\hline & \multicolumn{2}{|c|}{ Weg Eff } & \multicolumn{2}{c|}{ Bin Eff } \\
\hline & 1 removal & 2 removals & 1 removal & 2 removals \\
\hline$\mu$ & 0.0447567 & 0.08679 & 0.00040613 & 0.00065 \\
\hline$\sigma$ & 0.047793 & 0.06677 & 0.000145 & 0.000212 \\
\hline
\end{tabular}

